\title{
Searches for supersymmetry in events with photons at CMS
}

\author{
Johannes Schulz \\ on behalf of the CMS collaboration \\ RWTH Aachen University \\ E-mail: jschulz@cern.ch
}

Searches for new physics in events with one or more photons and missing transverse momentum produced in proton-proton collision data recorded by the CMS experiment during the CERN LHC Run2 operations (2016) are presented. The results of four searches are statistically combined and interpreted in the context of gauge-mediated supersymmetry models. A search for supersymmetry in events with at least one photon, missing transverse momentum, and large hadronic activity is presented, exploiting the b-jet multiplicity of the signal events.

XXIX International Symposium on Lepton Photon Interactions at High Energies - LeptonPhoton2019 August 5-10, 2019

Toronto, Canada

${ }^{*}$ Speaker. 


\section{Introduction}

The search for physics beyond the standard model is one of the key research topics at the CERN LHC. The extension of the standard model (SM) by supersymmetry (SUSY) provides a theory able to explain several shortcomings of the SM, e.g., the stabilization of the SM-like Higgs boson mass at the electroweak (EWK) scale and the origin of dark matter.

In gauge-mediated SUSY breaking (GMSB) scenarios, the gravitino $(\widetilde{\mathrm{G}})$ is the lightest SUSY particle (LSP). The lightest neutralino $\left(\widetilde{\chi}_{1}^{0}\right)$ is assumed to be the next-to-LSP (NLSP). In the signal scenarios considered here, R-Parity is assumed to be conserved, resulting in the pair production of SUSY particles and the stability of the LSP. The probability of the decay $\widetilde{\chi}_{1}^{0} \rightarrow \gamma+\widetilde{\mathrm{G}}$ depends on the mixture of the gauge eigenstates of the $\widetilde{\chi}_{1}^{0}$ and is at least $\cos ^{2}\left(\theta_{\mathrm{W}}\right)$ for bino-like $\widetilde{\chi}_{1}^{0}$, where $\theta_{\mathrm{W}}$ is the Weinberg angle, leading to an enhanced photon production. The $\widetilde{\mathrm{G}}$ escapes undetected, resulting in a significant imbalance in the measured transverse momentum sum.

The data-taking of the Run2 period of the CERN LHC at a center-of-mass energy of $13 \mathrm{TeV}$ is completed. First CMS results of searches for SUSY in final states with photons using the data set recorded in 2016 have already been published $[1,2,3,4]$. The data set corresponds to $35.9 \mathrm{fb}^{-1}$. A statistical combination of these four searches is presented and the results are interpreted in new signal scenarios based on GMSB ("GMSB combination") [5]. Furthermore, a search for SUSY exploiting the b-jet multiplicity in events with photons, jets, and missing transverse momentum $\left(p_{\mathrm{T}}^{\text {miss }}\right)$ is presented in these proceedings ("Photon + (b-)jet") [6].

Figure 1 (a) shows some example diagrams of the signal scenarios used in the GMSB combination. The first three diagrams show possible processes based on EWK $\widetilde{\chi}_{1}^{ \pm} \widetilde{\chi}_{2}^{0}$ and $\widetilde{\chi}_{1}^{ \pm} \widetilde{\chi}_{1}^{\mp}$ production, the fourth diagram illustrates a scenario based on gluino pair production. Figure 1 (b) shows the diagrams considered in the Photon $+(b-)$ jet analysis which are all based on strong gluino and top-squark production.

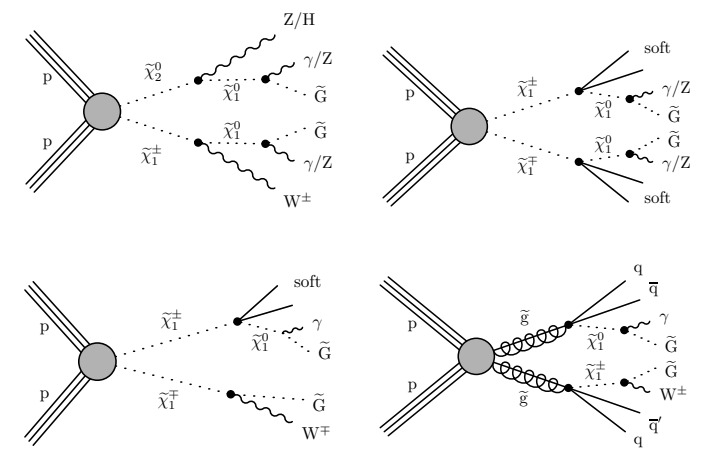

(a) GMSB combination diagrams

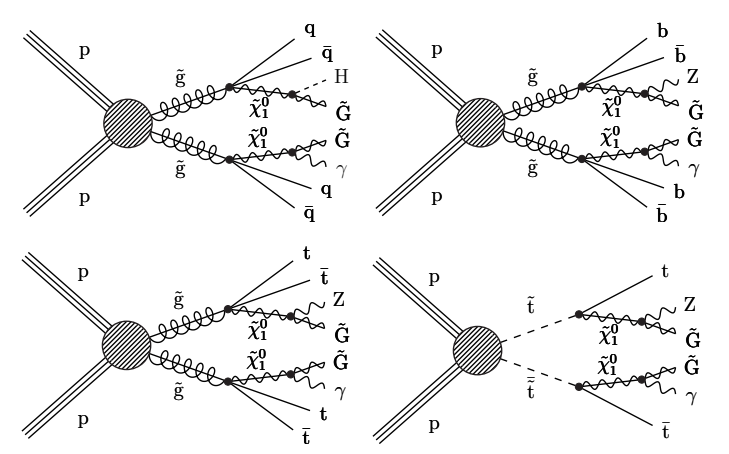

(b) Photon $+($ b-)jet diagrams

Figure 1: Example diagrams of the signal scenarios considered in the GMSB combination (a) and the Photon $+($ b-)jet analysis (b).

\section{GMSB combination}

The GMSB combination [5] is a statistical combination of four individual SUSY searches analyzing final states with at least one photon and large $p_{\mathrm{T}}^{\mathrm{miss}}$, namely the Photon $+\mathrm{S}_{\mathrm{T}}^{\gamma}[1]$, Pho- 
ton $+\mathrm{H}_{\mathrm{T}}^{\gamma}$ [2], Photon + Lepton [3], and Diphoton [4] analysis. While the Photon $+\mathrm{S}_{\mathrm{T}}^{\gamma}$ analysis preserves sensitivity to a large phase space by only exploiting photon and $p_{\mathrm{T}}^{\text {miss }}$ properties, the other three analyses apply more restrictive requirements to the event kinematics. The Photon $+\mathrm{H}_{\mathrm{T}}^{\gamma}$ analysis additionally requires a large hadronic activity, while the Photon + Lepton and Diphoton searches require the existence of one charged electron or muon and a second photon, respectively. The signal regions used in the combination are based on the signal selections of the individual searches with additional vetoes to ensure exclusiveness. The signal selections of the Photon + Lepton and Diphoton analysis are orthogonal due to the charged lepton veto applied in the Diphoton analysis. The Photon + Lepton analysis defines 36 signal regions binned in $p_{\mathrm{T}}^{\text {miss }}, E_{\mathrm{T}}^{\gamma}$, and the hadronic activity, while the signal regions in the Diphoton analysis are defined by six intervals in $p_{\mathrm{T}}^{\text {miss }}$. For the combination, events with electrons, muons, or a second photon are vetoed from the Photon $+\mathrm{S}_{\mathrm{T}}^{\gamma}$ and Photon $+\mathrm{H}_{\mathrm{T}}^{\gamma}$ signal regions. Events with $\mathrm{H}_{\mathrm{T}}^{\gamma}>2 \mathrm{TeV}$, where $\mathrm{H}_{\mathrm{T}}^{\gamma}$ is the scalar sum of the highest-energy photon and the $p_{\mathrm{T}}$ of all jets, are vetoed from the Photon $+\mathrm{S}_{\mathrm{T}}^{\gamma}$ category, adding three more signal regions to the combination binned in $p_{\mathrm{T}}^{\text {miss }}$. Events with $\mathrm{H}_{\mathrm{T}}^{\gamma}<2 \mathrm{TeV}$ are vetoed from the Photon $+\mathrm{H}_{\mathrm{T}}^{\gamma}$ category and binned in four intervals of $\mathrm{S}_{\mathrm{T}}^{\gamma}$, where $\mathrm{S}_{\mathrm{T}}^{\gamma}$ is the scalar sum of the photonic energy reconstructed in the event and $p_{\mathrm{T}}^{\text {miss }}$, resulting in a total of 49 signal regions defined in the combination, as shown in Fig. 2 (left).

The interpretation of these results in a full GMSB-based scenario is shown in Fig. 2 (middle). The scenario probes EWK gaugino production, with gluinos and squarks assumed to be too heavy to be produced. The expected and observed $95 \%$ confidence level (CL) upper exclusion limit for the combination and the individual searches are shown. The expected limit on Chargino masses is improved by around $100 \mathrm{GeV}$ compared to the individual searches. Figure 2 (right) shows the NLSP mass exclusion limits in an EWK simplified model in dependency of the branching fraction of the decay $\widetilde{\chi}_{1}^{0} \rightarrow \gamma+\widetilde{\mathrm{G}}$. The expected limit of the combination almost coincides with the expected limit of the Photon $+\mathrm{S}_{\mathrm{T}}^{\gamma}$ analysis and NLSP masses are excluded below $1200 \mathrm{GeV}$ for a branching fraction of $100 \%$ and below $1000 \mathrm{GeV}$ for $50 \%$.
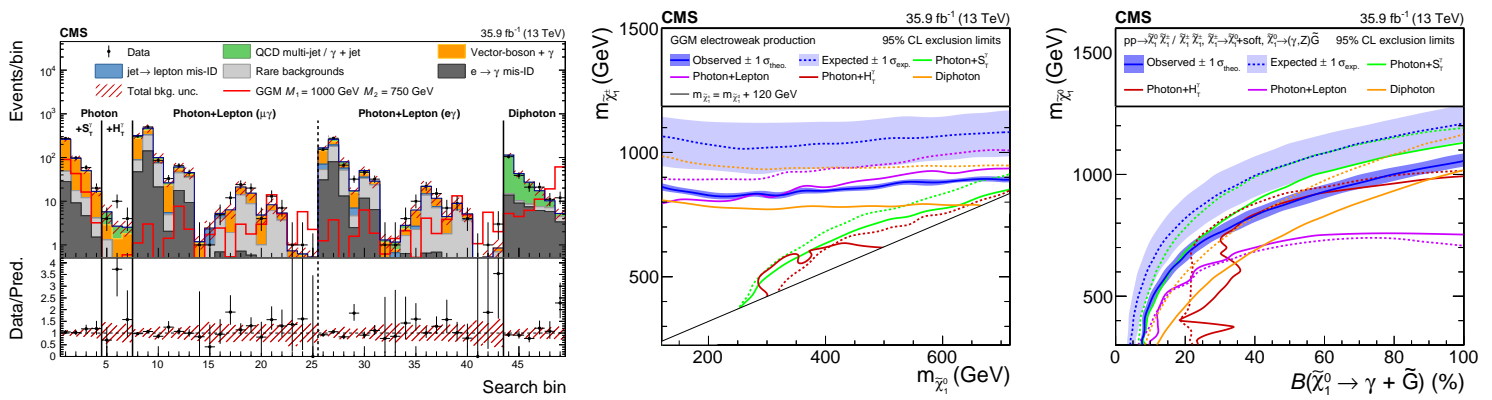

Figure 2: Predicted and observed events yields of the 49 signal regions used in the GMSB combination (left), expected and observed exclusion limits in a full GMSB-based scenario (middle), and a simplified model with varying branching fractions of the decay $\widetilde{\chi}_{1}^{0} \rightarrow \gamma+\widetilde{\mathrm{G}}$ (right), both assuming EWK production.

\section{Photon $+($ b-)jet analysis}

The Photon + (b-)jet analysis [6] selects events with at least one photon, large $p_{\mathrm{T}}^{\text {miss }}$, and a large $\mathrm{H}_{\mathrm{T}}^{\gamma}$. The events are further categorized by the b-jet multiplicity of the event to increase sensitivity 
to signal scenarios with an enhanced b-quark production.

To meet the requirements of the trigger, events are required to fulfill $\mathrm{H}_{\mathrm{T}}^{\gamma}>800 \mathrm{GeV}$ and $E_{\mathrm{T}}^{\gamma}>$ $100 \mathrm{GeV}$ or $\mathrm{H}_{\mathrm{T}}^{\gamma}>500 \mathrm{GeV}$ and $E_{\mathrm{T}}^{\gamma}>190 \mathrm{GeV}$, with at least two jets reconstructed in the event. Events must fulfill $p_{\mathrm{T}}^{\text {miss }}>100 \mathrm{GeV}$ and $\mid \Delta \phi$ (two highest- $p_{\mathrm{T}}$ jets, $\left.p_{\mathrm{T}}^{\text {miss }}\right) \mid>0.3$. Events with charged leptons are vetoed. The signal regions are divided into events with $2-4,5-6$, and at least 7 jets for both zero and at least one reconstructed b-jet. These six categories are further divided into events with $200<p_{\mathrm{T}}^{\text {miss }}<270 \mathrm{GeV}, 270<p_{\mathrm{T}}^{\text {miss }}<350 \mathrm{GeV}, 350<p_{\mathrm{T}}^{\text {miss }}<450 \mathrm{GeV}$, and $p_{\mathrm{T}}^{\text {miss }}>450 \mathrm{GeV}$. In the category with $2-4$ jets and zero b-jets, the highest $p_{\mathrm{T}}^{\text {miss }}$ bin is further subdivided into two intervals defined by $450<p_{\mathrm{T}}^{\text {miss }}<750 \mathrm{GeV}$ and $p_{\mathrm{T}}^{\text {miss }}>750 \mathrm{GeV}$, resulting in a total of 25 search regions. The contribution of the SM backgrounds varies across the signal regions and is mainly estimated using data control regions.

Figure 3 (left) shows the predicted and observed event yields in the 25 signal regions. No significant discrepancies between prediction and measurement are observed. 95\% CL upper cross section limits are set in simplified models based on gluino, Figure 3 (middle), and top-squark pair production, Figure 3 (right). Gluino masses up to $2120 \mathrm{GeV}$ and top-squark masses up to $1230 \mathrm{GeV}$ are excluded by the Photon $+(\mathrm{b}-)$ jet analysis.
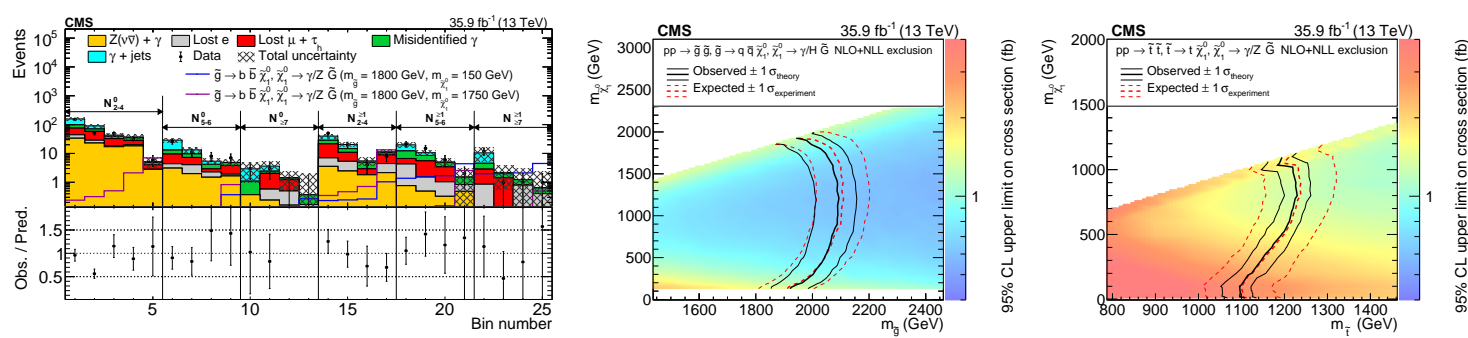

Figure 3: Yields of the 25 signal regions used in the Photon + (b-)jet analysis (left), expected and observed exclusion limits in a simplified model based on gluino (middle) and top-squark (right) pair production.

\section{References}

[1] CMS Collaboration, Search for gauge-mediated supersymmetry in events with at least one photon and missing transverse momentum in pp collisions at $\sqrt{s}=13 \mathrm{TeV}$, Physics Letters B 780 (2018) 118 [hep-ex/1711.08008].

[2] CMS Collaboration, Search for supersymmetry in events with at least one photon, missing transverse momentum, and large transverse event activity in proton-proton collisions at $\sqrt{s}=13 \mathrm{TeV}, \mathrm{JHEP} \mathbf{1 2}$ (2017) 142 [hep-ex/1707.06193].

[3] CMS Collaboration, Search for supersymmetry in final states with photons and missing transverse momentum in proton-proton collisions at 13 TeV, JHEP 06 (2019) 143 [hep-ex/1903.07070].

[4] CMS Collaboration, Search for supersymmetry in events with a photon, a lepton, and missing transverse momentum in proton-proton collisions at $\sqrt{s}=13 \mathrm{TeV}$, JHEP 01 (2019) 154.

[5] CMS Collaboration, Combined search for supersymmetry with photons in proton-proton collisions at $\sqrt{s}=13 \mathrm{TeV}$, Submitted to Physics Letters B, hep-ex/1907.00857v1.

[6] CMS Collaboration, Search for supersymmetry in events with a photon, jets, b-jets, and missing transverse momentum in proton-proton collisions at 13 TeV, Eur. Phys. J. C 79 (2019) 444. 